\title{
STEADY STATE SOLUTIONS OF THE SMOLUCHOWSKI EQUATION FOR RIGID NEMATIC POLYMERS UNDER IMPOSED FIELDS*
}

\author{
QI WANG ${ }^{\dagger}$, SARTHOK SIRCAR ${ }^{\ddagger}$, AND HONG ZHOU
}

\begin{abstract}
We solve the Smoluchowski equation for steady state solutions of rigid nematic polymers and suspensions under imposed elongational flow, magnetic or electric fields, respectively. Under the three imposed fields, we show that (1) the Smoluchowski equation can be cast into a generic form, (2) the external field must parallel to one of the eigenvectors of the second moment tensor in steady states, and (3) the steady state solution of the Smoluchowski equation (probability density function or simply pdf) is of the Boltzmann type parameterized by material parameters and two order parameters governed by two algebraic-integral equations. Then, we present a complete bifurcation diagram of the order parameters with respect to the material parameters by solving the algebraic-integral equations. The stability of the pdf solutions is inferred from the minimum of the free energy density. The solution method is extended to dilute solutions of dipolar, rigid nematic polymers under an imposed electric field. The first moment of the steady state pdf is shown to be parallel to the external field direction at sufficiently strong permanent dipole or relatively weak dipole-dipole interaction. In this case, the steady solution of the Smoluchowski equation is parameterized by one order parameter and material parameters in the Boltzmann form. Otherwise, the first moment is not necessarily parallel to the external field direction.
\end{abstract} field.

Key words. Liquid crystals, kinetic theory, polymers, elongational flow, magnetic field, electric

AMS subject classifications. 35A99, 35C15, 76W05, 82D60

\section{Introduction}

The kinetic theory is an effective tool in modeling soft matter and complex fluids $[25,3,20]$. In the past, it has been perceived as a sophisticated formalism hardly amenable to analytical analysis. Given the rising interest in mathematical analysis on kinetic theories recently, various attempts have been made to analyze the properties of the partial differential equations in kinetic theories and obtain their solutions analytically, semi-analytically and/or numerically $[4,26,8,9,10,11,12,13,19,14,16,15,17,18]$.

In this paper, we give a systematic derivation of the exact steady state solution of the Smoluchowski equation in semi-implicit form using a projection method and then study its variation with respect to material parameters numerically. We demonstrate the method with two examples. One is the concentrated solution of rigid nematic polymers in the elongational flow, or magnetic field, or electric field. The other is the dilute solution of dipolar, rigid nematic polymers under the influence of the electric field. In both cases, we give the exact solution in the semi-implicit form of an exponential function parameterized by order parameters governed by algebraicintegral equations. To our best knowledge, the idea was first used in the work of Ramalingam and Armstrong in a Doi type kinetic theory $[1,2]$ when solving the solution for the uniaxial order parameter in an imposed elongational flow. The basic idea stems from the fact that the solution of the Smoluchowski equation in potential

\footnotetext{
*Received: July 20, 2005; accepted (in revised version): October 21, 2005. Communicated by Pingwen Zhang.

${ }^{\dagger}$ Department of Mathematics, Florida State University, Tallahassee, FL 32306, USA, and School of Mathematics, Nankai University, Tianjin, 300071, P. R. China.

${ }^{\ddagger}$ Department of Mathematics, Florida State University, Tallahassee, FL 32306, USA.

$\S$ Department of Applied Mathematics, Naval Postgraduate School, Monterey, CA 93943, USA.
} 
fields is of the Boltzmann type, which is completely determined by the total potential. The total potential in turn can be parameterized by a set of scalar order parameters and prescribed field parameters. Once the order parameters are determined through a particular representation, their governing equations emerge in the form of algebraicintegral equations. In principle, this approach extends to any potentials that can be expressed as a finite sum of the linear combination of the spherical harmonic functions on the sphere.

\section{Steady states under an imposed elongational flow, electric or mag-} netic field

We adopt the extended Doi-Hess model for solutions of rigid nematic polymers $[20,21,22,23,24]$ with the well-known Maier-Saupe excluded volume potential

$$
V_{i}(\mathbf{m}, \mathbf{x}, t)=-\frac{3 N k T}{2}\langle\mathbf{m m}\rangle: \mathbf{m m},
$$

where $N$ is the dimensionless number density of the nematic polymer, $\mathbf{m}$ is a unit vector for the axis of symmetry of the molecule, which is modeled as a spheroid, $\langle\mathbf{m m}\rangle=\int_{\|\mathbf{m}\|=1} \mathbf{m m} f(\mathbf{m}, t) d \mathbf{m}$ is the second moment of $\mathbf{m}$ with respect to the probability density function $f(\mathbf{m}, t)[20,23]$. When the molecule (or nematic particle in the case of suspensions) is under an imposed electric or magnetic field, an induced dipole or magnetic moment in the molecule will occur even though it does not have an intrinsic dipole (nondipolar) or magnetic moment (nonferromagnetic). The potential due to the external field effect is given by the potential

$$
V_{H}=-\frac{\chi_{\alpha}}{2}(\mathbf{H} \cdot \mathbf{m})^{2}, V_{E}=-\frac{\alpha}{2}(\mathbf{E} \cdot \mathbf{m})^{2},
$$

respectively, where $\mathbf{H}$ is the magnetic field vector, and $\chi_{\alpha}$ is the difference of the susceptibility parallel and perpendicular to the molecular direction; $\mathbf{E}$ is the electric field, and $\alpha$ is the difference between the polarizability parallel and perpendicular to the molecular direction. We note that, in this formulation, the mean-field dipoledipole interaction due to the induced dipole is not accounted for.

The transport equation for the probability distribution function of the molecular orientation in monodomains is given by the Smoluchowski equation:

$$
\begin{aligned}
\frac{d f}{d t} & =\mathcal{R} \cdot\left[f \mathcal{R} \mu_{t}\right]-\mathcal{R} \cdot[\mathbf{m} \times \dot{\mathbf{m}} f], \\
\dot{\mathbf{m}} & =\mathbf{W} \cdot \mathbf{m}+a[\mathbf{D} \cdot \mathbf{m}-\mathbf{D}: \mathbf{m m m}],
\end{aligned}
$$

where time is nondimensionalized by a constant rotary diffusivity $D_{r}, \mathcal{R}=\mathbf{m} \times \frac{\partial}{\partial \mathbf{m}}$ is the rotational gradient operator, and $\frac{d}{d t}(\bullet)$ denotes the material derivative: $\frac{\partial}{\partial t}(\cdot)+$ $\mathbf{v} \cdot \nabla(\cdot), \mathbf{D}$ and $\mathbf{W}$ are the rate of strain tensor and vorticity tensor, respectively; $a$ is a geometry or shape parameter defined by $a=\frac{r^{2}-1}{r^{2}+1}$ with the molecular aspect ratio $r . \quad \mu=\ln f+\frac{1}{k T} V_{i}$ is the normalized chemical potential and $\mu_{t}=\mu+\frac{1}{k T} V_{H}$ or $\mu_{t}=\mu+\frac{1}{k T} V_{E}$ is the extended chemical potential including the normalized external potential.

For an elongational flow field stretching $(\gamma>0$, uniaxial elongation) or compressing $\left(\gamma<0\right.$, biaxial elongation) in the direction of $\mathbf{e}_{3}$, the velocity field is given by

$$
\mathbf{v}=-\frac{\gamma}{2}\left(x \mathbf{e}_{1}+y \mathbf{e}_{2}\right)+\gamma z \mathbf{e}_{3}
$$


It can be cast in the form of a potential effectively [28],

$$
V_{e}=-\frac{3 a \gamma}{4} k T \mathbf{e}_{3} \mathbf{e}_{3}: \mathbf{m m}
$$

In fact,

$$
-\mathbf{m} \times \dot{\mathbf{m}}=\frac{1}{k T} \mathcal{R} V_{e}
$$

since $\mathbf{W}=0$ in elongational flow fields. Therefore, the rotary convective term in the Smoluchowski equation can be absorbed into the extended chemical potential in (2.3).

Now that the external potentials for the elongational flow, magnetic and electric field are identical in form, we next illustrate the solution method for Smoluchowski equation in the case of the elongational flow field only. In this case, the Smoluchowski equation is rewritten in the form

$$
\frac{d f}{d t}=\mathcal{R} \cdot\left[f \mathcal{R} \mu_{t}\right]
$$

where $\mu_{t}=\ln f+\frac{1}{k T}\left(V_{i}+V_{e}\right)$. The steady state solution of the equation is given by

$$
\mu_{t}=C
$$

leading to

$$
f=\frac{1}{Z} e^{-\frac{1}{k T}\left(V_{i}+V_{e}\right)},
$$

where $Z$ is the normalizing constant to ensure $\langle 1\rangle=1$ or the partition function. We denote

$$
\nu=\frac{a \gamma}{2}
$$

and name it the effective Peclet number. Then, the total potential is given by

$$
V=V_{i}+V_{e}=-\frac{3 k T}{2}\left[N\langle\mathbf{m m}\rangle+\nu \mathbf{e}_{3} \mathbf{e}_{3}\right]: \mathbf{m m}
$$

We adopt a general representation of the second moment $\langle\mathbf{m m}\rangle$

$$
\langle\mathbf{m m}\rangle=s(\mathbf{n n}-\mathbf{I} / 3)+\beta\left(\mathbf{n}_{\perp} \mathbf{n}_{\perp}-\mathbf{I} / 3\right)+\frac{\mathbf{I}}{3},
$$

where $s$ and $\beta$ are two order parameters and $\mathbf{n}$ and $\mathbf{n}_{\perp}$ are two eigenvectors of $\langle\mathbf{m m}\rangle$ [27]. It follows from (2.12) that

$$
\begin{aligned}
& s=2\left\langle(\mathbf{n} \cdot \mathbf{m})^{2}\right\rangle+\left\langle\left(\mathbf{n}_{\perp} \cdot \mathbf{m}\right)^{2}\right\rangle-1 \\
& \beta=\left\langle(\mathbf{n} \cdot \mathbf{m})^{2}\right\rangle+2\left\langle\left(\mathbf{n}_{\perp} \cdot \mathbf{m}\right)^{2}\right\rangle-1 .
\end{aligned}
$$

If we parameterize the vector $\mathbf{m}$ relative to the orthonormal frame $\left(\mathbf{n}, \mathbf{n}_{\perp}, \mathbf{n}^{*}\right)$ as follows

$$
\mathbf{m}=\cos \theta \mathbf{n}+\sin \theta \cos \phi \mathbf{n}_{\perp}+\sin \theta \sin \phi \mathbf{n}^{*},
$$


where $\mathbf{n}^{*}$ is the third orthonormal eigenvector of $\langle\mathbf{m m}\rangle$ besides $\mathbf{n}$ and $\mathbf{n}_{\perp}$, and set $\mathbf{e}_{3}=\mathbf{n}$, then

$$
f=\frac{1}{Z} e^{\frac{3 N}{2}\left[\left(s-\frac{\beta}{2}\right)\left(\cos ^{2} \theta-1 / 3\right)+\frac{\beta}{2} \sin ^{2} \theta \cos 2 \phi\right]+\frac{3 \nu}{2} \cos ^{2} \theta}
$$

with

$$
Z=Z(s, \beta)=\int_{\|\mathbf{m}\|=1} e^{\frac{3 N}{2}\left[\left(s-\frac{\beta}{2}\right)\left(\cos ^{2} \theta-\frac{1}{3}\right)+\frac{\beta}{2} \sin ^{2} \theta \cos 2 \phi\right]+\frac{3 \nu}{2} \cos ^{2} \theta} d \mathbf{m} .
$$

Substituting (2.15) into the formula of $s$ and $\beta$, we arrive at the governing system of equations for the order parameters.

$$
\begin{aligned}
\beta & =\int_{\|\mathbf{m}\|=1} \sin ^{2} \theta \cos 2 \phi f d \mathbf{m}, \\
s & =\int_{\|\mathbf{m}\|=1} \frac{1}{2}\left(3 \cos ^{2} \theta-1\right) f d \mathbf{m}+\frac{\beta}{2} .
\end{aligned}
$$

Using a change of variable, we rewrite the order parameters as follows

$$
\begin{aligned}
\beta & =4 \int_{0}^{\pi} \int_{0}^{1}\left(1-z^{2}\right) \cos 2 \phi f d z d \phi \\
& =\frac{1}{\tilde{Z}} \int_{0}^{1}\left(1-z^{2}\right) e^{3 / 2(N(s-\beta / 2)+\nu) z^{2}} I_{1}(\zeta) d z, \\
s & =4 \int_{0}^{\pi} \int_{0}^{1} P_{2}(z) f d z+\beta / 2, \\
& =\frac{1}{\tilde{Z}} \int_{0}^{1} P_{2}(z) e^{3 / 2(N(s-\beta / 2)+\nu) z^{2}} I_{0}(\zeta) d z+\beta / 2, \\
f & =\frac{1}{\tilde{Z}} e^{\frac{3 N}{2}\left[\left(s-\frac{\beta}{2}\right) z^{2}+\frac{\beta}{2}\left(1-z^{2}\right) \cos 2 \phi\right]+\frac{3 \nu}{2} z^{2}},
\end{aligned}
$$

where

$$
\tilde{Z}(s, \beta)=\int_{0}^{1} e^{\frac{3}{2}(N(s-\beta / 2)+\nu) z^{2}} I_{0}(\zeta) d z
$$

$P_{2}(z)=\frac{1}{2}\left(3 z^{2}-1\right)$ is the second order Legendre polynomial, and $\zeta=\frac{3 N \beta}{4}\left(1-z^{2}\right)$. In the above, we used the definition for the modified Bessel function of the first kind:

$$
\pi I_{n}(z)=\int_{0}^{\pi} e^{z \cos \phi} \cos n \phi d \phi, n=0, \cdots, \infty
$$

Noticing that $\beta=0$ is a solution of (2.17), we deduce the implicit equation governing the uniaxial order parameter s:

$$
\begin{aligned}
s & =\int_{\|\mathbf{m}\|=1} \frac{1}{2}\left(3 \cos ^{2} \theta-1\right) \frac{1}{Z(s, 0)} e^{\frac{3 N s}{2}\left(\cos ^{2} \theta-1 / 3\right)+\frac{3 \nu}{2} \cos ^{2} \theta} f d \mathbf{m} \\
& =\int_{0}^{1} P_{2}(z) e^{\frac{3}{2}(N s+\nu) z^{2}} d z /\left(\int_{0}^{1} e^{\frac{3}{2}(N s+\nu) z^{2}} d z\right) .
\end{aligned}
$$


The free energy density of the nematic polymer system is given by

$$
A[f]=\int_{\|\mathbf{m}\|=1}\left[k T \ln f+\frac{V_{i}}{2}+V_{e}\right] f d \mathbf{m} .
$$

From (2.9), we arrive at the free energy density at steady states:

$$
\begin{gathered}
A[f]=\int_{\|\mathbf{m}\|=1}\left[-k T \ln Z-\frac{V_{i}}{2}\right] f d \mathbf{m} \\
=-k T\left[\ln Z-\frac{N}{2}\left(s^{2}-s \beta+\beta^{2}\right)\right] .
\end{gathered}
$$

This formula will be used to infer the stability of the steady states. The stable steady state is the global minimum of the free energy density. The metastable steady state is a local minimum of the free energy, but not the global one.

2.1. Reduced symmetry. It is known that the Smoluchowski equation is invariant under the rotational transformation in $\mathrm{SO}(3)$ when flows and external field effects are absent [29]. Namely,

$$
\frac{d}{d t} f=\mathcal{R}_{n} \cdot\left[f \mathcal{R}_{n} \mu\right]
$$

where $f=f(\mathbf{n}, t), \mathcal{R}_{n}=\mathbf{n} \times \frac{\partial}{\partial \mathbf{n}}, \mathbf{n}=\mathbf{U} \cdot \mathbf{m}$ for any $\mathbf{U} \in S O(3)$. With the imposed elongational flow of axis of symmetry $\mathbf{e}_{3}$, we denote the rotational group in the plane transverse to the axis by $S O(2)=\left\{\mathbf{U} \mid \mathbf{U} \in S O(3), \mathbf{U} \cdot \mathbf{e}_{3}=\mathbf{e}_{3}\right\}$. Then, from the invariant property of the Smoluchowski equation, we deduce eq. (2.3) is invariant under $\mathrm{SO}(2)$ since

$$
\mu_{t}=-\frac{3 N k T}{2} \mathbf{m m}:\langle\mathbf{m m}\rangle-\frac{3 \nu k T}{2} \mathbf{e}_{3} \mathbf{e}_{3}: \mathbf{m m}=-\frac{3 N k T}{2} \mathbf{n n}:\langle\mathbf{n n}\rangle-\frac{3 \nu k T}{2} \mathbf{e}_{3} \mathbf{e}_{3}: \mathbf{n n}
$$

for $\mathbf{n}=\mathbf{U} \cdot \mathbf{m}$, where $\mathbf{U} \in S O(2)$. I.e., there exists a reduced symmetry in the Smoluchowski equation under the imposed field in the plane orthogonal to the field. Namely, if there exists a pdf solution of the Smoluchowski equation, there exists a family of pdf solutions parameterized by $\mathrm{SO}(2)$.

In the extended Doi-Hess kinetic theory, the geometric parameter $a$ can be exploited to model both rodlike $(a>0)$ and disklike $(a<0)$ molecules. Effectively, it is the effective Peclet number that matters in the steady state solution. When molecules are disklike, an uniaxial elongational flow $(\gamma>0)$ is effectively equivalent to a biaxial elongational flow of rodlike nematic polymers since they share the same effective Peclet number and vice versa. Given the asymmetric correspondence between the rodlike and disklike molecules in biaxial and uniaxial elongation, we will focus on the rodlike nematic polymer in the following $(a>0)$. The steady states of disklike nematic polymers can be obtained from the correspondence principle.

2.2. Uniaxial elongation. Stretching or uniaxial elongation for rodlike nematic polymers corresponds to $\nu>0$. The steady state solutions consist of up to three uniaxial steady states with their uniaxial directors aligned in the direction of the flow and a family of biaxial solutions at sufficiently high polymer concentration parameterized by $\mathrm{SO}(2)$. The bifurcation diagram of the uniaxial steady states with respect to the dimensionless concentration has been documented in [27]: there are 


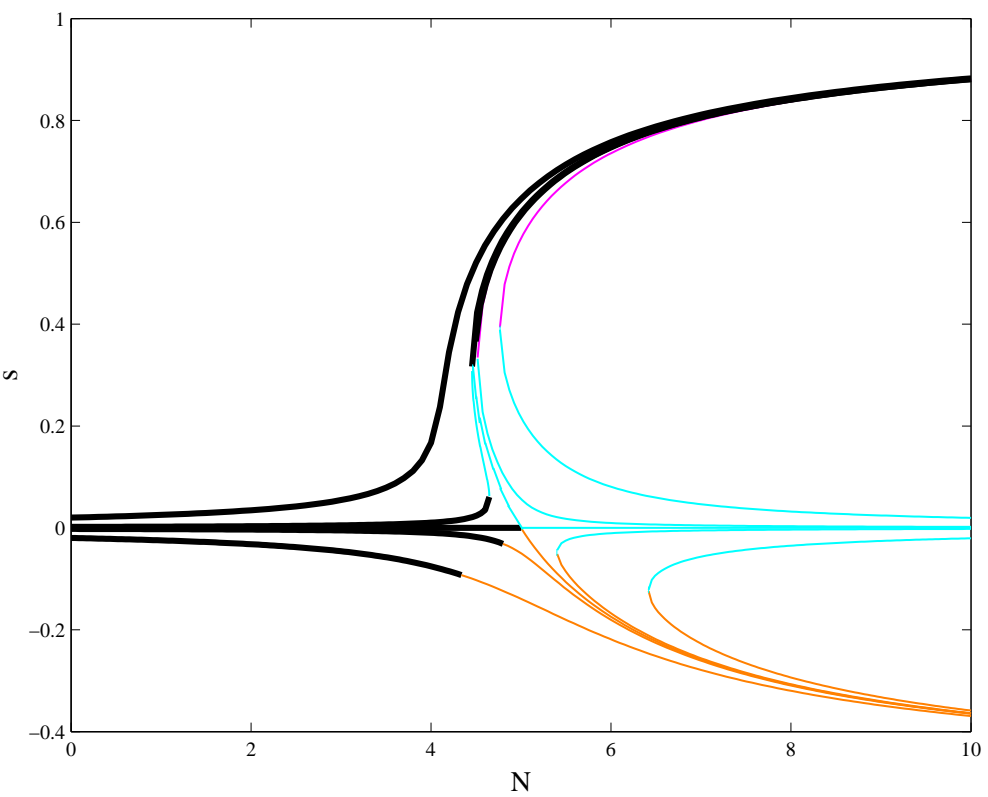

FIG. 2.1. The phase bifurcation diagram for the steady state uniaxial order parameter at $\nu=$ $-0.1,-0.01,0,0.01,0.1$ respectively. A bi-stability region exists for small magnitude Peclet numbers and in a small window of nematic polymer concentrations near the critical concentration $N=5$. If $\nu>0$, the highly aligned prolate state $(s>0)$ is stable while the less aligned prolate steady state is metastable; whereas the oblate state $(s<0)$ is stable up to certain critical concentration if $\nu<0$. The thick curves represent the stable branches while the thin curves depict the unstable ones.
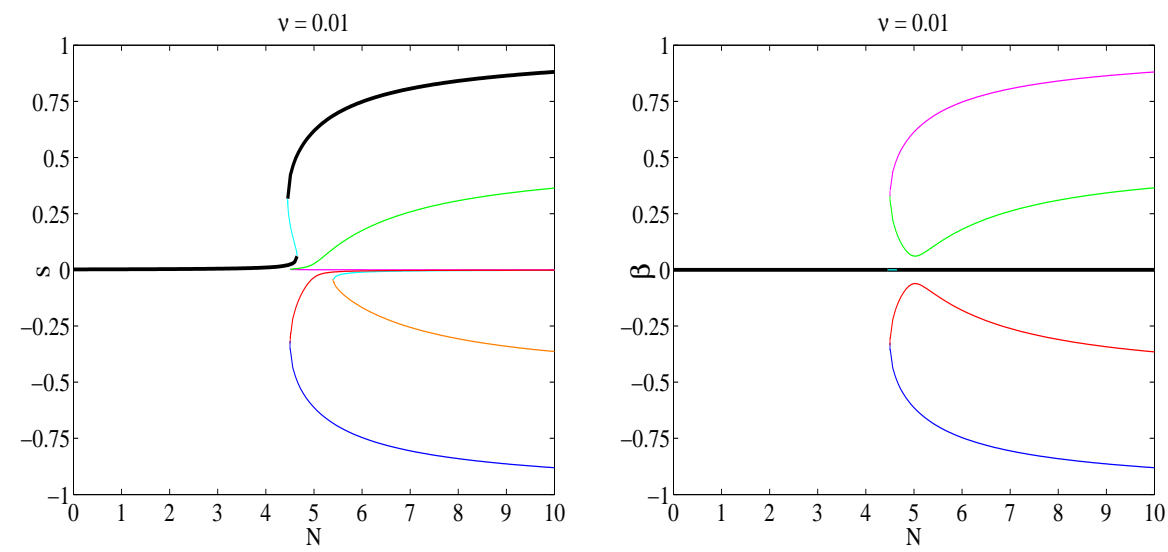

FIG. 2.2. The phase bifurcation diagram for all the steady state order parameters in uniaxial elongation. The stable solutions are uniaxial prolate ones $(s>0, \beta=0)$ of highly aligned and the less aligned. The biaxial states form a family of solutions parameterized by the rotational group SO(2), which are unstable. The Peclet number here is $\nu=0.01$. The thick curves depict the stable branches while the thin curves do the unstable ones.

up to two stable prolate steady states at small Peclet number regime limited to a small window of nematic polymer concentration and there is only one in the regime of large Peclet numbers. The highly aligned nematic steady state is always stable. 
When two stable prolate steady states coexist in a window of small Peclet numbers and nematic polymer concentrations the lesser aligned one is metastable. Figure 2.1 depicts the uniaxial steady state solutions in the phase space $(N, s)$, with $\nu=$ $-0.1,-0.01,0,0.01,0.1$, respectively.

The biaxial steady states emerged at sufficiently high concentration correspond to the entire nematic equilibrium family parameterized by $\mathrm{SO}(2)$ with their major directors aligned in the plane perpendicular to the direction of elongation absent of flow. However, these biaxial steady solutions are unstable. Figure 2.2 depicts all uniaxial steady states and a pair of biaxial steady state families with their major axes perpendicular to each other in the plane orthogonal to the direction of elongation.
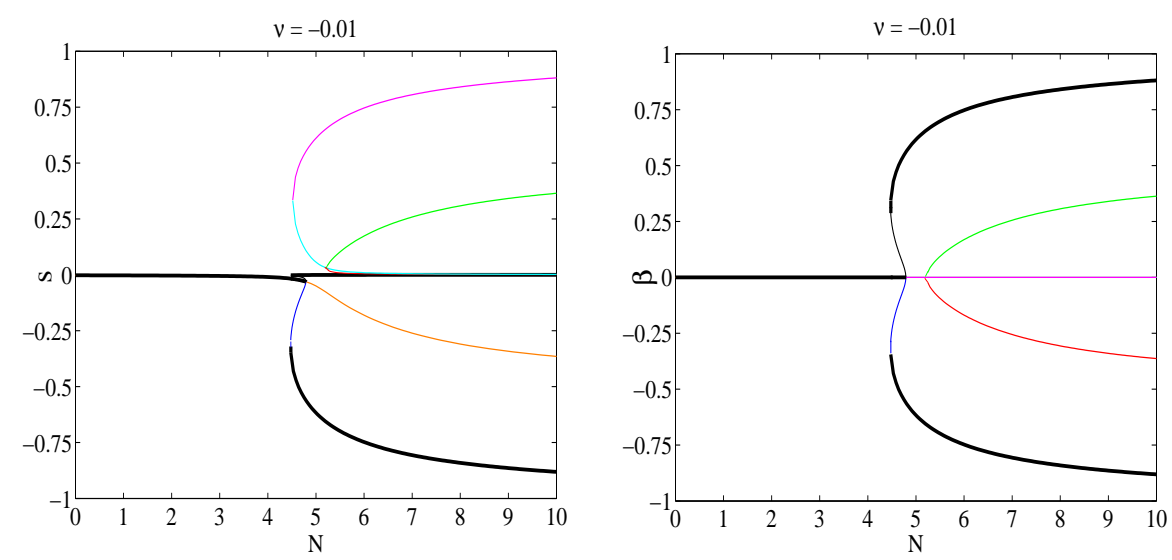

FIG. 2.3. The phase bifurcation diagram for all the steady state order parameters in biaxial elongation. The stable solution is the oblate $(s<0, \beta=0)$ one up to a critical concentration and then assumed by a family of biaxial solutions $(s \neq 0, \beta \neq 0)$. The bistability region may exist in a small window of nematic polymer concentrations at small Peclet number regimes. The Peclet number here is $\nu=-0.01$. The thick curves depict the stable branches while the thin curves do the unstable ones.

2.3. Biaxial elongation. When rodlike nematic polymers are in biaxial elongation, $\nu<0$, there exist up to three uniaxial steady states with their uniaxial directors aligned in the axis of the flow symmetry. At small concentration, the only uniaxial steady state is the oblate one; whereas there are two more prolate uniaxial steady states at sufficiently high concentration. At high enough concentration, a family of biaxial steady states parameterized by $\mathrm{SO}(2)$ emerges. At low concentration, the only stable steady state is the oblate uniaxial one. At sufficiently high concentration, a family of biaxial steady state parameterized by $\mathrm{SO}(2)$ is stable. The stable biaxial steady state is deformed from the prolate uniaxial equilibrium $(\nu=0)$ with their uniaxial director aligned in the plane orthogonal to the flow direction. Again, bi-stability may take place in the regime of small Peclet numbers for a limited range of concentration. Figure 2.3 depicts a representative bifurcation diagram for all steady states as functions of the Peclet number and dimensionless concentration.

We have obtained solutions of the Smoluchowski equation semi-analytically by assuming the imposed field parallels to one of the eigenvector direction of the second moment tensor. Next, we show that this is a fact.

To prove it, we note that the imposed field direction can be parameterized in the 
frame of $\mathbf{n}, \mathbf{n}_{\perp}, \mathbf{n}^{*}$ as follows:

$$
\mathbf{e}_{3}=\cos \theta^{\prime} \mathbf{n}+\sin \theta^{\prime} \cos \phi^{\prime} \mathbf{n}_{\perp}+\sin \theta^{\prime} \sin \phi^{\prime} \mathbf{n}^{*},
$$

where $\theta^{\prime}, \phi^{\prime}$ are constants. So,

$$
\mathbf{e}_{3} \cdot \mathbf{m}=\cos \theta \cos \theta^{\prime}+\sin \theta \sin \theta^{\prime} \cos \left(\phi-\phi^{\prime}\right) .
$$

The pdf solution of the Smoluchowski equation is given by

$$
f=\frac{1}{Z} e^{\left.\frac{3 N}{2}\left[\left(s-\frac{\beta}{2}\right)\left(\cos ^{2} \theta-1 / 3\right)+\frac{\beta}{2} \sin ^{2} \theta \cos 2 \phi\right]+\frac{3 \nu}{2}\left(\cos \theta \cos \theta^{\prime}+\sin \theta \sin \theta^{\prime} \cos \left(\phi-\phi^{\prime}\right)\right)^{2}\right]},
$$

where $Z$ is the normalizing constant. From the definition of the second moment equation, we arrive at three additional identities

$$
\mathbf{n} \cdot \mathbf{M} \cdot \mathbf{n}_{\perp}=0, \mathbf{n} \cdot \mathbf{M} \cdot \mathbf{n}^{*}=0, \mathbf{n}_{\perp} \cdot \mathbf{M} \cdot \mathbf{n}^{*}=0
$$

where $\mathbf{M}=\langle\mathbf{m m}\rangle$. Next, we show that either $\theta^{\prime}=0$ or $\theta^{\prime}=\pi / 2$ together with $\phi^{\prime}=0$ or $\phi^{\prime}=\pi / 2$. This is equivalent to say that $\mathbf{e}_{3}$ is in one of the eigenvector directions of the second moment tensor $\mathbf{M}$.

It follows from (2.29)

$$
\left\langle\cos \theta \sin \theta \cos \left(\phi-\phi^{\prime}\right)\right\rangle=0
$$

for any values of $\phi^{\prime}$. We define

$$
F(\lambda)=\int_{\|\mathbf{m}\|=1} \cos \theta \sin \theta \cos \left(\phi-\phi^{\prime}\right) e^{h} / Z d \mathbf{m},
$$

where

$$
\begin{gathered}
h(\lambda)=\frac{3 N}{2}\left[\left(s-\frac{\beta}{2}\right)\left(\cos ^{2} \theta-1 / 3\right)+\frac{\beta}{2} \sin ^{2} \theta \cos 2 \phi\right]+\frac{3 \nu}{2}\left(\left(\cos \theta \cos \theta^{\prime}\right)^{2}+\right. \\
\left.\left.\left(\sin \theta \sin \theta^{\prime} \cos \left(\phi-\phi^{\prime}\right)\right)^{2}+2 \lambda \cos \theta \cos \theta^{\prime} \sin \theta \sin \theta^{\prime} \cos \left(\phi-\phi^{\prime}\right)\right)\right] .
\end{gathered}
$$

We note that (2.30) implies

$$
F(1)=0
$$

Since the integrand in the integral of $F(0)$ is an analytical function of $\sin ^{2} \theta$ multiplied by $\cos \theta$ which is an odd function about $\theta=\pi / 2$, it can be easily shown to be zero.

However,

$$
F^{\prime}(\lambda)=\text { const } \times \int_{\|\mathbf{m}\|=1} \sin 2 \theta^{\prime} \sin ^{2} 2 \theta \cos ^{2}\left(\phi-\phi^{\prime}\right) e^{h} / Z d \mathbf{m} \neq 0
$$

provided

$$
\sin 2 \theta^{\prime} \neq 0
$$

This would contradict to the fact that $F(0)=F(1)=0$ if $(2.35)$ were true. Thus

$$
\theta^{\prime}=0, \pi / 2
$$


In the case of $\theta^{\prime}=\pi / 2$, we define

$$
G(\lambda)=\int_{\|\mathbf{m}\|=1} \sin ^{2} \theta \cos \phi \sin \phi e^{g} / Z d \mathbf{m},
$$

where

$$
\begin{aligned}
& g=\frac{3 N}{2}\left[\left(s-\frac{\beta}{2}\right)\left(\cos ^{2} \theta-1 / 3\right)+\frac{\beta}{2} \sin ^{2} \theta \cos 2 \phi\right]+\frac{3 \nu}{2} \sin ^{2} \theta\left(\cos ^{2} \phi \cos ^{2} \phi^{\prime}+\right. \\
& \left.\left.\sin ^{2} \phi \sin ^{2} \phi^{\prime}+2 \lambda \sin \phi \cos \phi \sin \phi^{\prime} \cos \phi^{\prime}\right)\right] .
\end{aligned}
$$

We recall that (2.29) implies $G(1)=0$. At $\lambda=0$, the integrand is given by an exponential function of $\cos 2 \phi$ multiplied by $\sin 2 \phi$. The integral in $\phi$ over $[0,2 \pi]$ is therefore equal to zero. I.e.,

$$
G(0)=0 .
$$

Then, using the same argument, we arrive at

$$
G^{\prime}(\lambda)=\text { const } \times \sin 2 \phi^{\prime} \int_{\|\mathbf{m}\|=1} \sin ^{4} \theta \sin ^{2} 2 \phi e^{g} / Z d \mathbf{m} \neq 0
$$

provided $\sin 2 \phi^{\prime} \neq 0$. This would contradict to $G(0)=G(1)=0$ if it were true. Hence, $\phi^{\prime}=0, \pi / 2$. We then conclude that $\mathbf{e}_{3}$ must be in one of the principal axes or eigenvector directions of the second moment tensor $\mathbf{M}$.

THEOREm 2.1. When the Smoluchowski equation with the Maier-Saupe excluded volume potential is driven under an imposed magnetic, or electric field, or an elongational flow field, one of the principal axes of the second moment of the steady state probability density function solution must be parallel to the imposed field direction.

\section{Effect of an imposed electric field on a dilute solution of nematic} polymers

We consider a dilute solution of dipolar, rigid nematic polymers or suspensions, where the excluded volume effect is neglected. When the electric field is applied, the total potential consisting of the intermolecular (dipole-dipole) and external electric potential is given by

$$
U=-\alpha\langle\mathbf{m}\rangle \cdot \mathbf{m}-\mu \mathbf{E} \cdot \mathbf{m}-\frac{\alpha_{0}}{2} \mathbf{E E}: \mathbf{m m},
$$

where $\alpha_{0}$ is the difference of the polarizability parallel and perpendicular to $\mathbf{m}, \mu$ is the strength of the permanent dipole and $\alpha$ is the strength of the intermolecular dipole-dipole interaction potential.

We set

$$
\langle\mathbf{m}\rangle=s_{1} \mathbf{q}_{1},\left\|\mathbf{q}_{1}\right\|=1
$$

where

$$
s_{1}=\left\langle\mathbf{q}_{1} \cdot \mathbf{m}\right\rangle
$$

is an order parameter describing the averaged molecular orientation about the direction $\mathbf{q}_{1}$. We extend $\mathbf{q}_{1}$ into an orthonormal basis $\mathbf{q}_{1}, \mathbf{q}_{2}$ and $\mathbf{q}_{3}$ and parameterize $\mathbf{m}$ and $\mathbf{E}$ with respect to the basis:

$$
\begin{aligned}
\mathbf{m} & =\cos \theta \mathbf{q}_{1}+\sin \theta \cos \phi \mathbf{q}_{2}+\sin \theta \sin \phi \mathbf{q}_{3}, \\
\mathbf{E} & =\|\mathbf{E}\|\left[\cos \theta^{\prime} \mathbf{q}_{\mathbf{1}}+\sin \theta^{\prime} \cos \phi^{\prime} \mathbf{q}_{\mathbf{2}}+\sin \theta^{\prime} \sin \phi^{\prime} \mathbf{q}_{\mathbf{3}}\right] .
\end{aligned}
$$


Assuming $\mathbf{E}|| \mathbf{q}_{1}$, we arrive at

$$
\begin{aligned}
& f=\frac{1}{Z} e^{\frac{1}{k T}\left[\left(\alpha s_{1}+\mu E\right) \cos \theta+\frac{\alpha_{0}}{2} E^{2} \cos ^{2} \theta\right]} \\
& s_{1}=\langle\cos \theta\rangle=\int_{-1}^{1} z e^{\frac{1}{k T}\left[\left(\alpha s_{1}+\mu E\right) z+\frac{\alpha_{0}}{2} E^{2} z^{2}\right]} d z / Z \\
& Z=\int_{-1}^{1} e^{\frac{1}{k T}\left[\left(\alpha s_{1}+\mu E\right) z+\frac{\alpha_{0}}{2} E^{2} z^{2}\right]} d z
\end{aligned}
$$

Figure 3.1 depicts the bifurcation diagram in the phase space $\left(\alpha, s_{1}\right)$ at selected values of $\mu, \alpha_{0}$ and $E=\|\mathbf{E}\|$. The stable branch is identified by examining the free energy density at steady state

$$
A=\int_{\|\mathbf{m}\|=1} k T(f \ln f)-\left[\frac{\alpha}{2}\langle\mathbf{m}\rangle \cdot \mathbf{m}+\mu \mathbf{m} \cdot \mathbf{E}+\frac{\alpha_{0}}{2} \mathbf{E E}: \mathbf{m m}\right] f d \mathbf{m}=\left[\frac{\alpha}{2} s_{1}^{2}-k T \ln Z\right] .
$$

At zero electric field strength, the order parameter goes through a second order phase transition as $\alpha$ increases. The critical strength is $\alpha=3$. When the electric field is applied, the symmetric phase diagram is broken so that a single branch of positive (negative) $s_{1}$ forms for all values of $\alpha$ and positive (negative) values of $E$ and two

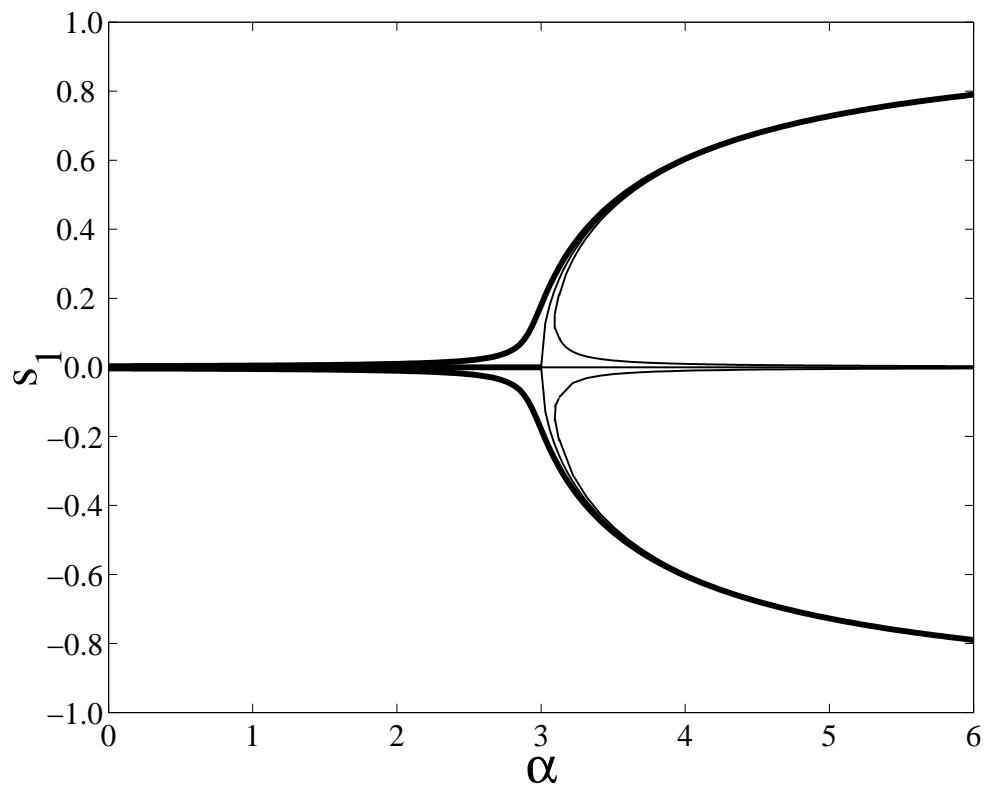

FIG. 3.1. The phase bifurcation diagram for the steady state order parameter $s_{1}$ as functions of $\alpha$. The parameter values are $k T=1, \alpha_{0}=0.2, \mu=0.1, E=-0.1,0,0.1$. The steady state bifurcation diagram is symmetric about $s_{1}=0$ and occurs at $\alpha_{c}=3$. When $E>0$, the symmetry is broken in such a way that a stable and positive order parameter exists for all $\alpha>0$ while a pair of negative order parameters emerge at sufficiently large values of $\alpha$. Whereas $E<0$, a stable negative order parameter persists for all $\alpha>0$ while a pair of positive order parameters emerge at sufficiently large values of $\alpha$. This indicates that nematic polymers incline to orient in the direction of the externally imposed electric field. The thick curves depict the stable branches while the thin curves do the unstable ones. 
branches of the order parameter $s_{1}$ of negative (positive) values emerge through a saddle node bifurcation. The single branch order parameter is stable, indicating that the averaged molecular orientation favors the direction of the external field.

We next show that $\mathbf{q}_{1}$ must be parallel to $\mathbf{E}$ under certain conditions, i.e., the orientational axis of the first moment is dictated by the external field. From the parametrization of the electric field, we have

$f=\frac{1}{Z} e^{\frac{1}{k T}\left[\left(\alpha s_{1}\right) \cos \theta+\mu E\left(\cos \theta \cos \theta^{\prime}+\sin \theta \sin \theta^{\prime} \cos \left(\phi-\phi^{\prime}\right)\right)+\frac{\alpha_{0}}{2} E^{2}\left(\cos \theta \cos \theta^{\prime}+\sin \theta \sin \theta^{\prime} \cos \left(\phi-\phi^{\prime}\right)\right)^{2}\right]}$,

where $Z$ is the normalizing constant. The other conditions that we can use are

$$
\mathbf{q}_{2} \cdot\langle\mathbf{m}\rangle=\mathbf{q}_{3} \cdot\langle\mathbf{m}\rangle=0
$$

This translates to

$$
\langle\sin \theta \sin \phi\rangle=\langle\sin \theta \cos \phi\rangle=0 .
$$

It follows from (3.9) that

$$
\langle\sin \theta \cos (\phi-\tilde{\phi})\rangle=0
$$

for any values of $\tilde{\phi}$.

THEOREM 3.1. When the solution of dipolar, rigid nematic polymers is driven by an imposed electric field, the first moment of the steady state probability density function must be parallel to the external field direction provided $|\mu| \geq\left|\alpha_{0} E\right|$.

Proof. We first assume $s_{1} \neq 0$ since the first moment is a zero vector otherwise. We set $\tilde{\phi}=\phi^{\prime}$ in (3.10) and define

$$
H=\int_{\|\mathbf{m}\|=1} \sin \theta \cos \left(\phi-\phi^{\prime}\right) e^{\psi} d \mathbf{m},
$$

where

$$
\begin{array}{r}
\psi=\frac{1}{k T}\left[\left(\alpha s_{1}\right) \cos \theta+\mu E\left(\cos \theta \cos \theta^{\prime}+\sin \theta \sin \theta^{\prime} \cos \left(\phi-\phi^{\prime}\right)\right)+\right. \\
\left.\frac{\alpha_{0}}{2} E^{2}\left(\cos \theta \cos \theta^{\prime}+\sin \theta \sin \theta^{\prime} \cos \left(\phi-\phi^{\prime}\right)\right)^{2}\right]
\end{array}
$$

We denote

$$
\begin{aligned}
& \psi=\frac{1}{k T}\left[\alpha s_{1} \cos \theta+\mu E \cos \theta \cos \theta^{\prime}+\frac{\alpha_{0}}{2} E \cos ^{2} \theta \cos ^{2} \theta^{\prime}+\right. \\
& \left.a(\theta) \cos \left(\phi-\phi^{\prime}\right)+b(\theta) \cos ^{2}\left(\phi-\phi^{\prime}\right)\right]
\end{aligned}
$$

where

$$
\begin{aligned}
& a(\theta)=\left(\mu E+\alpha_{0} E^{2} \cos \theta \cos \theta^{\prime}\right) \sin \theta \sin \theta^{\prime}, \\
& b(\theta)=\frac{\alpha_{0}}{2} E^{2} \sin ^{2} \theta \sin ^{2} \theta^{\prime} .
\end{aligned}
$$


Taking into account the periodicity of the trigonometric functions, we observe that $\mathrm{H}$ does not depend on $\phi^{\prime}$. Without loss of generality, we set $\phi^{\prime}=0$. Through a series of variable changes, we arrive at

$$
\begin{array}{r}
H=\int_{0}^{\pi / 2} \int_{0}^{\pi / 2} \sin ^{2} \theta \cos \phi e^{\frac{1}{k T}\left[\frac{\alpha_{0}}{2} E^{2} \cos ^{2} \theta \cos ^{2} \theta^{\prime}+b(\theta) \cos ^{2} \phi\right]}[\sinh (a(\theta) \cos \phi / k T) \\
\left.e^{\frac{1}{k T}\left[\alpha s_{1} \cos \theta+\mu E \cos \theta \cos \theta^{\prime}\right]}+\sinh (a(\pi-\theta) \cos \phi / k T) e^{\frac{-1}{k T}\left[\alpha s_{1} \cos \theta+\mu E \cos \theta \cos \theta^{\prime}\right]}\right] d \theta d \phi .
\end{array}
$$

If $|\mu| \geq\left|\alpha_{0} E\right|, a(\theta) a(\pi-\theta) \geq 0$. Thus, if $\sin \theta^{\prime} \neq 0, H>0$, which contradicts to $H=0$. This implies, $\sin \theta^{\prime}=0$. I.e., the magnetic field is parallel to the direction of the first moment $\mathbf{q}_{1}$.

The condition on the size of the parameters in theorem 2 is necessary for some values of $\alpha$. In fact, if $|\mu|<\left|\alpha_{0} E\right|$ and $\alpha$ is large enough, then the direction of $<\mathbf{m}>$ may be different from that of $\mathbf{E}$ (which will be shown below by numerical calculations). This result can be illustrated by an intuitive physical argument. Each rodlike polymer is subject to two potentials: 1) the external potential caused by the electric field and 2) the (mutual) intermolecular potential caused by other polymers in the ensemble. It is known that in the absence of the external potential, there is an I-N phase transition caused by the dipole-dipole interaction between rigid polymers rigid nematic when $\alpha>3 k T[14,15,18]$. In other words, for $\alpha>3 k T$, polymers tend to form a cluster with a distinguished direction (director). In the absence of the external potential, the director of the cluster is arbitrary. In the presence of the external potential, however, the director of the cluster is no longer arbitrary. If the director of the cluster is not a stationary point of the external potential, then the cluster cannot be a steady state solution. The external potential has at least two stationary points for any values of $\mu, \alpha_{0}, \mathbf{E}$. To continue the discussion, we need to switch to a spherical system different from the one used above. We select the $z$-axis as the direction of $\mathbf{E}$ and the $y$-axis perpendicular to the plane spanned by $<\mathbf{m}>$ and $\mathbf{E}$ (assuming $<\mathbf{m}>$ and $\mathbf{E}$ are not parallel to each other of course). In this coordinate system,

$$
\mathbf{E}=E(0,0,1),<\mathbf{m}>=\left(r_{1}, 0, r_{3}\right)
$$

In spherical coordinates, the external potential is given by

$$
U_{E x t}(\theta, \phi)=-\mu E \cos \theta-\frac{\alpha_{0}}{2} E^{2} \cos ^{2} \theta=-\alpha_{0} E^{2}\left(\frac{\mu}{\alpha_{0} E} \cos \theta+\frac{1}{2} \cos ^{2} \theta\right) .
$$

When $|\mu|<\left|\alpha_{0} E\right|$, the external potential has a third stationary point, $\theta_{0}$, determined by

$$
\cos \left(\theta_{0}\right)=-\frac{\mu}{\alpha_{0} E}
$$

The stationary point $\theta_{0}$ is between 0 and $\pi$. Therefore, the intuitive analysis indicates that when $|\mu|<\left|\alpha_{0} E\right|$ and $\alpha$ large, there can be a steady state cluster whose director is different from the direction of $\mathbf{E}$.

We next prove that when $0<|\mu|<\left|\alpha_{0} E\right|$, there is a critical value $\alpha^{*}$ such that for $\alpha \leq \alpha^{*}$, all steady state solutions satisfy that $\left\langle\mathbf{m}>\right.$ is parallel to $\mathbf{E}$ (i.e. $r_{1}=0$ ). For $\alpha>\alpha^{*}$, we show numerically that there exists a steady state solution where $\langle\mathbf{m}>$ is not parallel to $\mathbf{E}$ (i.e. $\left.r_{1} \neq 0\right)$. 
In the Cartesian coordinate system with the direction of $\mathbf{E}$ as the $z$-axis:

$$
\begin{aligned}
\mathbf{m} & =\left(m_{1}, m_{2}, m_{3}\right), \mathbf{E}=E(0,0,1),<\mathbf{m}>=\left(r_{1}, 0, r_{3}\right) \\
U(\mathbf{m}) & =-\alpha r_{1} m_{1}-\left(\mu E+\alpha r_{3}\right) m_{3}-\frac{\alpha_{0}}{2} E^{2} m_{3}^{2} \\
f(\mathbf{m}) & =\frac{\exp \left(\frac{1}{k T}\left[\alpha r_{1} m_{1}+\left(\mu E+\alpha r_{3}\right) m_{3}+\frac{\alpha_{0}}{2} E^{2} m_{3}^{2}\right]\right)}{\int_{\|\mathbf{m}\|=\mathbf{1}} \exp \left(\frac{1}{k T}\left[\alpha r_{1} m_{1}+\left(\mu E+\alpha r_{3}\right) m_{3}+\frac{\alpha_{0}}{2} E^{2} m_{3}^{2}\right]\right) d \mathbf{m}} .
\end{aligned}
$$

In the spherical coordinate system:

$$
\begin{aligned}
\mathbf{m} & =(\sin \theta \cos \phi, \sin \theta \sin \phi, \cos \theta) \\
U(\theta, \phi) & =-\alpha r_{1} \sin \theta \cos \phi-\left(\mu E+\alpha r_{3}\right) \cos \theta-\frac{\alpha_{0}}{2} E^{2} \cos ^{2} \theta \\
f(\theta, \phi) & =\frac{\exp \left(\frac{1}{k T}\left[\alpha r_{1} \sin \theta \cos \phi+\left(\mu E+\alpha r_{3}\right) \cos \theta+\frac{\alpha_{0}}{2} E^{2} \cos ^{2} \theta\right]\right)}{\int_{0}^{\pi} \int_{0}^{2 \pi} \exp \left(\frac{1}{k T}\left[\alpha r_{1} \sin \theta \cos \phi+\left(\mu E+\alpha r_{3}\right) \cos \theta+\frac{\alpha_{0}}{2} E^{2} \cos ^{2} \theta\right]\right) d \phi \sin \theta d \theta} .
\end{aligned}
$$

The nonlinear integral equations governing $r_{1}$ and $r_{3}$ are

$$
\begin{aligned}
& r_{1}=\int_{0}^{\pi} \int_{0}^{2 \pi} \sin \theta \cos \phi f(\theta, \phi) d \phi \sin \theta d \theta \\
& r_{3}=\int_{0}^{\pi} \int_{0}^{2 \pi} \cos \theta f(\theta, \phi) d \phi \sin \theta d \theta
\end{aligned}
$$

THEOREM 3.2. When $|\mu|<\left|\alpha_{0} E\right|$, there exists a critical $\alpha^{*}$ such that all steady solutions of (3.21) satisfy $r_{1}=0$ if $\alpha<\alpha^{*}$.

We first prove that all solutions of (3.21) satisfy $r_{1}=0$ if $\alpha<k T$. We prove it by contradiction. Suppose there is a solution of (3.21) satisfying $r_{1} \neq 0$.

In the probability density $f(\theta, \phi)$ above, we replace $r_{1}$ by $r$ and treat it as a variable. We consider the function

$$
F_{1}(r)=r-<\sin \theta \cos \phi>,
$$

which satisfies

$$
F_{1}(0)=F_{1}\left(r_{1}\right)=0 .
$$

The derivative of $f(\theta, \phi)$ with respect to $r$ is

$$
\frac{d f(\theta, \phi)}{d r}=\frac{\alpha}{k T}(\sin \theta \cos \phi-<\sin \theta \cos \phi>) F_{1}(\theta, \phi) .
$$

The derivative of $F_{1}(r)$ is

$$
\begin{aligned}
& \frac{d F_{1}(r)}{d r}=1-\frac{\alpha}{k T}<\sin \theta \cos \phi(\sin \theta \cos \phi-<\sin \theta \cos \phi>)> \\
& =1-\frac{\alpha}{k T} \operatorname{Var}(\sin \theta \cos \phi)>\left\{\begin{array}{ll}
1, & \alpha \leq 0 \\
1-\left\langle\sin ^{2} \theta \cos ^{2} \phi\right\rangle, & 0<\alpha<k T,
\end{array}\right\}>0,
\end{aligned}
$$

where Var denotes the variance. Clearly, $\frac{d F_{1}}{d r}>0$ when $\alpha<k T$, which contradicts (3.23). Thus, the only solution for $r_{1}$ is zero when $\alpha<k T$. Let

$$
\alpha^{*}=\inf \left\{\alpha \mid \text { eq. }(3.21) \text { has a solution with } r_{1} \neq 0\right\} \text {. }
$$




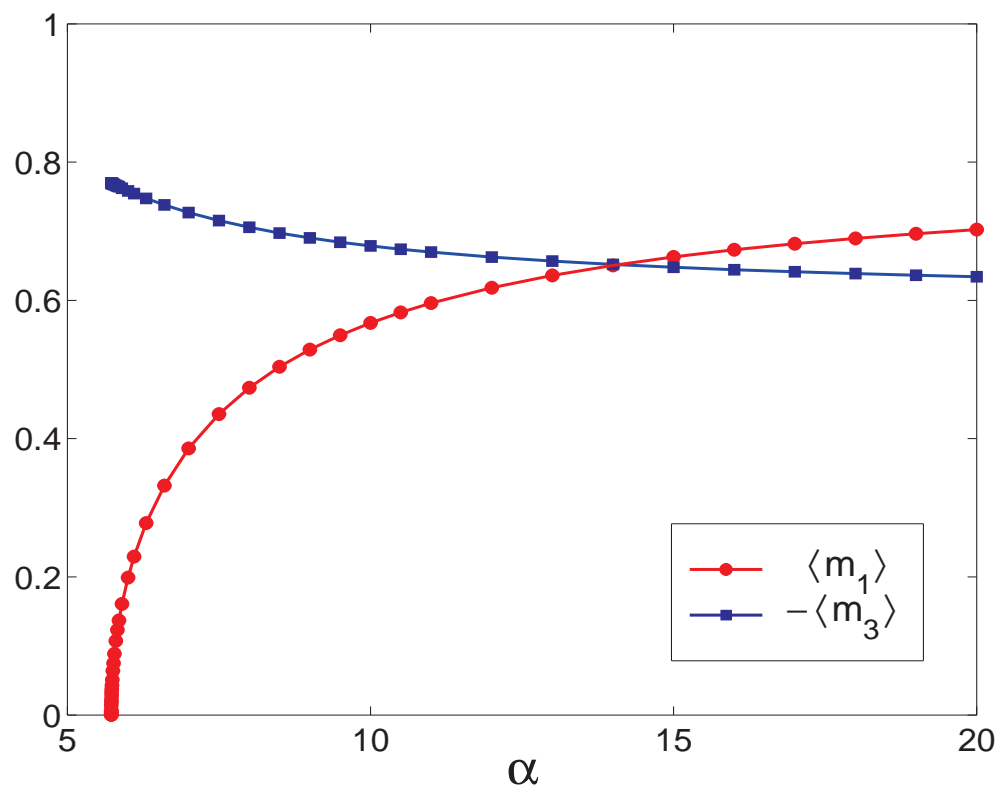

FIG. 3.2. The plot of $\left\langle m_{1}\right\rangle$ (i.e. $r_{1}$ ) and $\left\langle m_{3}\right\rangle$ (i.e. $-r_{3}$ ) as functions of $\alpha$ with $k T=1$. It shows the existence of the first moment that is not parallel to any eigenvectors of the second moment.

Clearly, $\alpha^{*}$ exists and $\alpha^{*} \geq k T$. Then, $r_{1}=0$ is the only solution if $\alpha<\alpha^{*}$.

Figure 3.2 depicts the steady state solution whose director is not parallel to $\mathbf{E}$. In Figure 3.2, < $m_{1}>$ (i.e. $r_{1}$ ) and $-<m_{3}>$ (i.e. $-r_{3}$ ) are shown as functions of $\alpha$. The parameters used here are $\mu=0.6 k T, \alpha_{0}=k T$, and $E=1$. For this set of parameters $\alpha^{*} \approx 5.7226567 k T, k T=1$.

3.1. Reduced symmetry. The direction of the first moment is arbitrary in equilibrium. However, it is no longer arbitrary when the electric field is imposed. When the first moment is parallel to the external field direction, for instance when $\alpha<\alpha^{*}$ and $|\mu|<\alpha_{0} E \mid$ or when $|\mu|>\left|\alpha_{0} E\right|$, the solution is invariant with respect to the rotational group $\mathrm{SO}(2)$ defined in the previous section. Otherwise, the direction of the first moment is well-defined and the pdf solution of the Smoluchowski equation may no longer be invariant under $\mathrm{SO}(2)$.

\section{Conclusion}

We have demonstrated the projection method for solving the Smoluchowski equation with the Maier-Saupe potential for rigid nematic polymers and the dipole-dipole interaction potential for dipolar rigid nematic polymers coupled with external fields. The method is general and can be used to solve the Smoluchsowki equation with any potential that is a function of the finite sum of spherical harmonic functions plus the external potential. The solution is always of the Boltzmann type and parameterized by a finite set of order parameters. The stability of the pdf solution can be inferred from the free energy density function within the order parameter space.

Acknowledgment. Efforts sponsored by the Air Force Office of Scientific Research, Air Force Materials Command, USAF, under grant number F49550-05-10025, and the National Science Foundation through grant DMS-0204243 are gratefully 
acknowledged. H. Zhou was partially supported by the Naval Postgraduate School Research Initiation Program.

\section{REFERENCES}

[1] S. Ramalingam and R. C. Armstrong, Analysis of isothermal spinning of liquid crystalline polymers, J. Rheology, 37 (6), 1141-1169, 1993.

[2] A. V. Bhave, R. K. Menon, R. C. Armstrong and R. A. Brown, A constitutive equation for liquid crystalline polymer solutions, J. Rheology, 37, 413-441, 1993.

[3] R. G. Larson, The Rheology of Complex Fluids, Oxford University Press, New York, 1998.

[4] R. G. Larson and H. C. Ottinger, Effect of molecular elasticity on out-of-plane orientations in shearing flows of liquid-crystalline polymers, Macromolecules, 24, 6270-6282, 1991.

[5] S. Chandrasekhar, Liquid Crystals, 2nd ed., Cambridge University Press, Cambridge, 1992.

[6] P. G. de Gennes and J. Prost, The Physics of Liquid Crystals, 2nd ed., Oxford University Press, New York, 1993.

[7] F. M. Leslie, Theory of flow phenomena in liquid crystals, Advances in Liquid Crystals, 4, 1-81, 1979.

[8] M. G. Forest, R. Zhou and Q. Wang, Kinetic structure simulations of nematic polymers in plane Couette cells, I: The algorithm and benchmarks, SIAM MMS, 3(4), 853-870, 2005.

[9] M. G. Forest, R. Zhou and Q. Wang, Kinetic structure simulations of nematic polymers in plane Couette cells, II, SIAM MMS, in press, 2005.

[10] M. G. Forest, R. Zhou and Q. Wang, Chaotic boundaries of nematic polymers in mixed shear and extensional flows, Physical Review Letters, 93 (8), 088301-088305, 2004.

[11] P. Constantin, I. Kevrekidis and E. S. Titi, Asymptotic states of a Smoluchowski equation, Arch. Rat. Mech. Anal., 174, 365-384, 2004.

[12] P. Constantin, I. Kevrekidis and E.S. Titi, Remarks on a Smoluchowski equation, Discrete and Continuous Dynamical Systems, 11, 101-112, 2004.

[13] P. Constantin and J. Vukadinovic, Note on the number of steady states for a 2D Smoluchowski equation, Nonlinearity, 18, 441-443, 2005.

[14] I. Fatkullin and V. Slastikov, Critical points of the Onsager functional on a sphere, Nonlinearity, 18, 2565-2580, 2005.

[15] H. Liu, H. Zhang and P. Zhang, Axial symmetry and classification of stationary solutions of Doi-Onsager equation on the sphere with Maier-Saupe potential, Comm. Math. Sci., 3, 201-218, 2005.

[16] C. Luo, H. Zhang and P. Zhang, The structure of equilibrium solution of $1 D$ Smoluchowski equation, Nonlinearity, 18, 379-389, 2005.

[17] B. Jourdain, T. Lelievre and C. LeBris, Existence of solution for a micro-macro model of polymeric fluid: the FENE model, Journal of Functional Analysis, 209, 162-193, 2004.

[18] H. Zhou, H. Wang, M. G. Forest and Q. Wang, A new proof on axisymmetric equilibria of a three-dimensional Smoluchowski equation, Nonlinearity, 18, 2815-2825, 2005.

[19] W. E, T. Li and P. Zhang Well-posedness for the dumbbell model of polymeric fluids, Comm. Math. Phys., 248, 409-427, 2004.

[20] M. Doi and S. F. Edwards, The Theory of Polymer Dynamics, Clarendon Press, Oxford, 1986.

[21] S. Hess, Fokker-Planck-equation approach to flow alignment in liquid crystals, Z Naturforschung 31a, 1034-1037, 1976

[22] N. Kuzuu and M. Doi, Constitutive equations for nematic liquid crystals under weak velocity gradients derived from a molecular kinetic equation, J. Phys. Soc. of Japan, 52, 3486-3494, 1983.

[23] Q. Wang, A hydrodynamic theory of nematic liquid crystalline polymers of different configurations, J. Chem. Phys., 116, 9120-9136, 2002.

[24] Q. Wang, M. G. Forest and R. Zhou, A hydrodynamic theory for solutions of nonhomogeneous nematic liquid crystalline polymers with density variations, J. Fluid Eng., 126, 180-188, 2004.

[25] B. Bird, R. C. Armstrong and O. Hassager, Dynamics of Polymeric Liquids, I, II, John Wiley and Sons, New York, 1987.

[26] M. G. Forest, R. Zhou and Q. Wang, The weak shear phase diagram for nematic polymers, Rheological Acta, 43(1), 17-37, 2004.

[27] Q. Wang, Comparative studies on closure approximations in flows of liquid crystal polymers, I. Elongational flows, J. of Non-Newtonian Fluid Mech., 72, 141-162, 1997. 
[28] H. See, M. Doi and R. G. Larson, The effect of steady flow fields on the isotropic ematic phase transition of rigid rod-like polymers, J. Chem. Phy., 92(1), 792-800, 1990.

[29] M. G. Forest, Q. Wang, R. Zhou and E. Coate, Monodomain response of arbitrary aspect ratio nematic polymers in general linear planar flows, J. of Non-Newtonian Fluid Mechanics, 118(1), 17-31, 2004. 